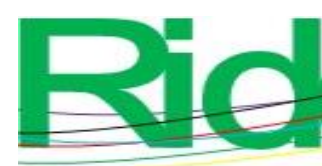

\title{
Modelo bifactorial de equilibrio moral emocional en estudiantes universitarios
}

\author{
Bifactorial Model of Emotional Moral Balance in University Students
}

\begin{abstract}
Modelo bifatorial de equilíbrio moral emocional em estudantes universitários

Víctor Hugo Robles Francia Universidad Juárez Autónoma de Tabasco, México vicrob13@yahoo.com.mx https://orcid.org/0000-0003-1046-4768
\end{abstract}

\section{Resumen}

El objetivo del presente trabajo fue validar y confirmar el ajuste de un modelo bifactorial de equilibrio moral emocional (EME) considerando la culpa y el orgullo como factores determinantes. Para la validez del modelo, se realizó un análisis factorial exploratorio (AFE) con 177 participantes, que se ratificó con un análisis factorial confirmatorio (AFC) con 300 personas. En el AFE se encontró una estructura de dos factores correspondientes a la culpa y el orgullo. El AFC validó el ajuste del modelo, el constructo de EME, con dos variables latentes, la culpa y el orgullo, y un total de 12 variables observables.

Palabras clave: análisis factorial, balance emocional, culpa, orgullo. 


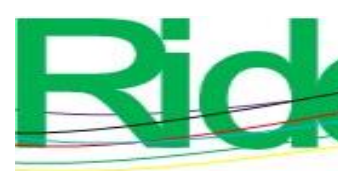

Revista Iberoamericana para la Investigación y el Desarrollo Educativo ISSN $2007-7467$

pocas investigaciones han examinado la relación entre los estándares morales y los factores emocionales morales del comportamiento de las personas (Tangney et al., 2007).

Al respecto, Beruchashvili, Price y Gentry (2006) aclaran que el nivel de moralidad aceptado representa una base de equilibrio moral en términos de fortalezas y debilidades morales. De acuerdo con Horne y Powell (2016), el individuo sustenta sobre esta base sus pensamientos, elecciones y acciones, de conformidad a las normas sociales interiorizadas. Estos mismos autores aseguran que, desde el punto de vista del equilibrio moral, el individuo hace lo posible para compensar alguna desviación en su comportamiento y así, alinearlo a las normas sociales para balancear su armonía moral.

Conforme a Cova et al. (2015) y Greene y Haidt (2002), las emociones, además de ser ventanas para comprender lo que se debe hacer, también son ballestas que impulsan la realización de lo que es correcto. Así pues, las emociones morales son aquellas acaecidas por el cabal cumplimiento o incumplimiento de los intereses sociales, de las finalidades de alguna persona que se reconoce como autoridad moral u originadas por el acatamiento o no del deber moral con uno mismo (Cova et al., 2015).

Asimismo, Bericart (2012) Vélez y Ostrosky (2006) argumentan que las emociones morales negativas, manifestadas en algún tipo de aflicción, y las positivas, que originan cierta sensación gratificante, se balancean tratando de establecer un equilibrio moral. En relación con las emociones morales negativas y positivas, Tangney et al. (2007) y Kaplan y Tivnan (2014) señalan que la culpa es una emoción fundamental negativa, surgida de alguna violación de los estándares morales, y que se manifiesta por una sensación dolorosa; y que el orgullo es otra emoción elemental, pero, a diferencia de la culpa, esta es positiva y trae a quien la experimenta una sensación grata. Estas dos emociones morales son primarias, comúnmente adaptativas y autoconscientes, que no dependen de ninguna otra emoción previa (Prinz, 2007). Sin embargo, estas emociones pueden tener una autoevaluación implícita o explícita y ser o no advertidas conscientemente por el ser humano (Kaplan y Tivnan, 2014; Tangney et al., 2007).

Rebega (2017) enfatiza en que la culpa hace sufrir a la persona y el orgullo le produce un disfrute. Puede afirmarse, apoyados en Tangney et al. (2007) y Kaplan y Tivnan (2014), que la culpa es una emoción ampliamente adaptativa, que implica una autopunición que le causa sufrimiento a quien la padece (Freud, 1923). Este sufrimiento en la persona debido a la culpa es ocasionado por haber infringido las normas sociales y no cumplir la moral autoimpuesta que guía su conducta. De esta manera, la persona como agente, que actúa y trae 


\section{Materiales y método}

La investigación tuvo un alcance descriptivo-correlacional. Las variables fueron descritas y se analizó la incidencia e interrelación entre ellas. Estuvo orientada a identificar la asociación de las variables culpa y orgullo mediante patrones predecibles para una población determinada. Contó con un diseño no experimental transversal, puesto que las variables fueron analizadas tal como se comportan en el contexto estudiado, sin intervención alguna. Fue de tipo transversal, debido a que la recolección de datos se realizó en un solo periodo de tiempo (Hernández, Fernández, Baptista, 2014).

Se realizaron dos estudios. En el primero se llevó a cabo un AFE y en el segundo, un AFC. Ambos con muestras independientes con el fin de explorar y confirmar el número de factores de la Escala de Equilibrio Moral Emocional (EEME). Se realizaron también las pruebas de confiabilidad y de validez del instrumento.

El primer estudio, el AFE, consideró 177 participantes: estudiantes universitarios de diferentes carreras de un instituto tecnológico superior del sureste de México, con una edad promedio de 23.89 años (DE = 6.5), 123 femeninos y 54 masculinos. Fueron seleccionados mediante un muestreo no probabilístico por conveniencia (Etikan, Abubakar y Sunusi, 2016). Lo anterior de acuerdo con Hair, Black, Babin y Anderson (2014), quienes sugieren un tamaño de muestra mínima de 10 observaciones por cada ítem. La EEME tiene 12 ítems, por lo que se cumplió inclusive con un mayor número de participantes por ítem (14.75).

La captura de datos y el análisis estadístico se realizó con el software estadístico SPSS versión 23. El AFE consistió en la prueba de Bartlett y Kaiser-Meyer-Olkin (KMO) y Bartlett y el análisis varimax para obtener los componentes principales de la EEME. Asimismo, se realizaron las pruebas de confiabilidad mediante el cálculo del alfa de Cronbach y la de validez convergente y discriminante. Para la validez, se analizaron las correlaciones mediante el coeficiente $r$ de Pearson entre las diferentes variables observadas, ítems, y las variables latentes, culpa y orgullo; las correlaciones positivas fueron consideradas evidencia de validez convergente, mientras que las correlaciones negativas y la ausencia de correlación fueron consideradas evidencia de validez discriminante.

En el segundo estudio se realizó un AFC, que examinó 300 participantes: estudiantes universitarios de seis distintas universidades mexicanas, de 19.8 años en promedio (DE = 3.25), 189 mujeres, 111 hombres; así, al igual que con el estudio anterior, se cumplió con el tamaño de muestra sugerido (Hair et al., 2014). Estos participantes contestaron la EEME de 


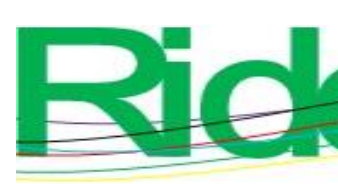

Revista Iberoamericana para la Investigación y el Desarrollo Educativo ISSN $2007-7467$

forma presencial, bajo los mismos lineamientos que en el primer estudio. Se empleó el software AMOS, complementando el software SPSS versión 23, para confirmar la estructura factorial a través del modelado de ecuaciones estructurales de la EEME. Igualmente, se ejecutó la prueba de confiabilidad de alfa de Cronbach y el análisis de correlaciones para la validez convergente y discriminante.

\section{Instrumento}

Los participantes contestaron la EEME (anexo 1), diseñada por Robles (2019), correspondiente al modelo bifactorial de culpa y orgullo, que evalúa la consistencia reactiva a partir de la sensación de horror de la lectura de un parricidio y de dos emociones morales básicas, la culpa y el orgullo. La culpa en función del grado de la sensación repugnante y el orgullo por la sensación placentera de autoimágenes atractivas percibidas. Así, los sujetos juzgan cada una de las aseveraciones sobre el afecto agradable del orgullo, lo que permite evaluar la relación entre la estructura cognitiva y la emotiva del juicio moral.

La lectura de la historia de horror moral, un parricidio cometido por el protagonista, denominado Juan, estimula en el espectador una reacción emocional, culpa u orgullo (Prinz, 2006, 2007). El estímulo del sujeto, bajo la interrogante “¿Si tú fueras Juan, en qué grado sentirías culpa (u orgullo) por matar a tu padre?", se registra en 12 ítems, seis sobre culpa y seis sobre orgullo, correspondientes a los estadios morales kohlbergianos (Kohlberg, 1992; Kohlberg y Hersh, 1977). Estos en una escala tipo Likert de 1 a 10, donde 1 implica no sentir nada y 10 indica sentir completamente la emoción. El EME se cuantifica mediante la diferencia de la suma de los ítems de culpa menos la suma de los respectivos del orgullo.

La EEME se aplicó de forma presencial, en formato impreso, en el lugar de los participantes; allí se les explicó el objetivo de las encuestas y la escala de evaluación. Se les solicitó su consentimiento informado y se aclaró que las respuestas serían tratadas de forma confidencial; también se les pidió que meditaran las respuestas y se instó a que manifestaran su sensación lo más fielmente posible. 


\section{Resultados}

\section{Estudio 1}

\section{Análisis factorial exploratorio}

Para la validez del modelo se realizó primeramente un AFE, el cual requirió previamente una medida de adecuación muestral de KMO apropiada (Ferrando y Anguiano, 2010). Además, el resultado de la prueba de esfericidad de Bartlett fue estadísticamente significativo, por lo que se demostró suficiente grado de relación entre los ítems del instrumento para ser analizados factorialmente (tabla 1).

Tabla 1. Prueba KMO y esfericidad de Bartlett

\begin{tabular}{|l|l|l|}
\hline \multicolumn{2}{|l|}{ Medida de adecuación muestral de KMO } & 0.818 \\
\hline $\begin{array}{l}\text { Prueba de esfericidad de } \\
\text { Bartlett }\end{array}$ & Ji al cuadrado aproximado & 1265.750 \\
\cline { 2 - 3 } & Gl & 66 \\
\cline { 2 - 3 } & Sig. & 0.000 \\
\hline
\end{tabular}

Fuente: Elaboración propia

El análisis de varianza total explicada indicó que los dos componentes, correspondientes al factor de culpa y orgullo, tienen varianzas mayores que uno, y entre los dos componentes explicaron más de $65 \%$ del total de la varianza total (tabla 2).

Tabla 2. Varianza total explicada

\begin{tabular}{|l|l|l|l|}
\hline & \multicolumn{3}{|c|}{ Suma de rotación de cargas cuadradas } \\
\hline Componente & Total & $\begin{array}{l}\% \text { de } \\
\text { Varianza }\end{array}$ & \begin{tabular}{l} 
Acumulativo \\
\hline 1
\end{tabular} \\
\hline 2 & 4.023 & 33.528 & 33.528 \\
\hline
\end{tabular}

Fuente: Elaboración propia

El análisis del componente principal demostró que los ítems se agruparon alrededor de dos componentes. En el componente uno, el factor de orgullo, se agruparon seis ítems; y en el segundo componente, el de culpa, se agruparon los seis ítems restantes (tabla 3). 
Tabla 3. Matriz de componentes rotados

\begin{tabular}{|c|c|c|c|}
\hline \multirow[t]{2}{*}{ Pregunta } & \multirow[t]{2}{*}{ Clave } & \multicolumn{2}{|c|}{ Componente } \\
\hline & & 1 & 2 \\
\hline $\begin{array}{l}\text { 1) Solo por quitarle la vida a alguien y quizás por } \\
\text { defenderme vaya a prisión. }\end{array}$ & Met-jc 1 & & 0.641 \\
\hline $\begin{array}{l}\text { 2) Por matar a mi padre, yo no podría ver a mi } \\
\text { madre a los ojos. }\end{array}$ & Met-jc 2 & & 0.781 \\
\hline $\begin{array}{l}\text { 3) Por matar a mi padre me señalarían, además de } \\
\text { mi madre, toda mi familia, mi novia y amigos. }\end{array}$ & Met-jc 3 & & 0.872 \\
\hline $\begin{array}{l}\text { 4) Porque me atormentaría imaginar que las } \\
\text { personas que más amo no pudieran vivir en armonía } \\
\text { en sociedad y menos junto a mí por mi culpa. }\end{array}$ & Met-jc 4 & & 0.884 \\
\hline $\begin{array}{l}\text { 5) Que traería el rechazo de la gente y de toda la } \\
\text { sociedad por ser el asesino de su padre. }\end{array}$ & Met-jc 5 & & 0.856 \\
\hline $\begin{array}{l}\text { 6) Porque no cumplí el principio inculcado por mi } \\
\text { madre, el amor a la vida, la prudencia y el respeto } \\
\text { hacia el padre. }\end{array}$ & Met-jc 6 & & 0.710 \\
\hline $\begin{array}{l}\text { 7) Por defender mi vida y emplear lo que aprendí de } \\
\text { defensa personal. }\end{array}$ & Met-jo 1 & 0.803 & \\
\hline $\begin{array}{l}\text { 8) Por defenderme bien, cuidar de mí, como me lo } \\
\text { enseñó mi mamá. }\end{array}$ & Met-jo 2 & 0.784 & \\
\hline $\begin{array}{l}\text { 9) Por matar a mi padre, quien no se ocupó de mí } \\
\text { nunca y violó a mi mamá, que siendo ladrón tendría } \\
\text { la aprobación de la gente y cualquiera actuaría de la } \\
\text { misma forma. }\end{array}$ & Met-jo 3 & 0.800 & \\
\hline $\begin{array}{l}\text { 10) Porque aparte de mi madre, toda mi familia y la } \\
\text { sociedad me reconocerían. }\end{array}$ & Met-jo 4 & 0.848 & \\
\hline $\begin{array}{l}\text { 11) Que traería el reconocimiento de la gente y de } \\
\text { toda lo sociedad por castigar a alguien que no } \\
\text { cumple con la reglas sociales ni humanas }\end{array}$ & Met-jo 5 & 0.838 & \\
\hline
\end{tabular}




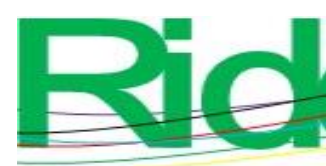

Revista Iberoamericana para la Investigación y el Desarrollo Educativo ISSN 2007 - 7467

\begin{tabular}{|c|c|c|}
\hline $\begin{array}{l}\text { 12) Porque cumplí el principio de salvaguardar mi } \\
\text { vida, principio inculcado por mi madre, el de justicia } \\
\text { y el respeto del deber. }\end{array}$ & Met-jo 6 & 0.824 \\
\hline
\end{tabular}

Fuente: Elaboración propia

\section{Índice de confiabilidad}

Se evaluó la consistencia interna de la EEME utilizando la prueba estadística de alfa de Cronbach, cuyo resultado fue aceptable, de acuerdo con lo sugerido por Nunnally (1970), quien señala que un valor aceptable para esta prueba debe ser superior a 0.70. Asimismo, cada una de las dos dimensiones, culpa y orgullo, arrojó valores superiores a 0.70. Estos resultados indicaron que la EEME y sus dos factores tienen una buena confiabilidad para la muestra poblacional (tabla 4).

Tabla 4. Análisis de confiabilidad

\begin{tabular}{|l|l|l|}
\hline Escala y factor & Alfa de Cronbach & Ítems \\
\hline EME & 0.777 & 12 \\
\hline Culpa & 0.882 & 6 \\
\hline Orgullo & 0.902 & 6 \\
\hline
\end{tabular}

Fuente: Elaboración propia

\section{Validez convergente y discriminante}

Para el análisis de la validez de la EEME, se realizó la prueba de correlación de Pearson. Al realizar el análisis de correlaciones entre los ítems de la escala, se obtuvieron valores entre 0.395 y .729, lo que indica que las variables comparten una varianza mínima.

Respecto de la evidencia de validez convergente en la tabla 5, se encontraron correlaciones significativas y positivas. Los seis ítems de culpa tuvieron correlaciones positivas altas (entre 0.658 y 0.872 ) con el factor de culpa y los seis de orgullo igualmente correlacionaron altamente (entre 0.799 y 0.8538 ) con el factor de orgullo. En relación con la evidencia de validez discriminante, se encontraron correlaciones negativas y débiles (de 0.022 a -0.146) entre los ítems de culpa y el factor de orgullo, de la misma forma que los seis ítems de orgullo correlacionaron negativa y débilmente con el de culpa (de -0.026 a - 0.195). 
Tabla 5. Correlación de Pearson entre ítems y factores

\begin{tabular}{|l|l|l|}
\hline & Culpa & Orgullo \\
\hline Met-jc 1 & $0.658^{* *}$ & -0.096 \\
\hline Met-jc 2 & $0.781 * *$ & -0.022 \\
\hline Met-jc 3 & $0.861 * *$ & -0.090 \\
\hline Met-jc 4 & $0.872 * *$ & -0.093 \\
\hline Met-jc 5 & $0.855^{* *}$ & -0.146 \\
\hline Met-jc 6 & $0.732 * *$ & -0.097 \\
\hline Met-jo 1 & -0.138 & $0.812 * *$ \\
\hline Met-jo 2 & $-0.195 * *$ & $0.803 * *$ \\
\hline Met-jo 3 & 0.026 & $0.799 * *$ \\
\hline Met-jo 4 & -0.032 & $0.838^{* *}$ \\
\hline Met-jo 5 & -0.045 & $0.824 * *$ \\
\hline Met-jo 6 & $-0.149 *$ & $0.833 * *$ \\
\hline
\end{tabular}

Fuente: Elaboración propia

\section{Estudio 2}

\section{Análisis factorial confirmatorio}

El estudio 2, mediante el AFC, evaluó el modelo bifactorial de la EEMO, que reportó los índices de ajuste que se muestran en la tabla 6; el valor de la raíz del error cuadrático medio RMSEA fue cercano a 0.06, considerado entonces óptimo, y el residuo estandarizado cuadrático medio SRMR fue cercano a 0.05 , por lo que se calificó como un buen ajuste (Cangur y Ercan, 2015; Hu y Bentler, 1999). Complementariamente, el índice de ajuste comparativo (CFI), el índice de Tucker-Lewis (TLI) y el índice de bondad de ajuste (GFI) fueron superiores a 0.95, lo que confirma un ajuste óptimo (Cupani, 2012; Medrano y Muñoz, 2017). Por último, otro índice que ratificó el modelo fue el ji al cuadrado entre grados de libertad, cuyo resultado fue menor a cinco, un valor aceptable (Chión y Charles, 2016; Escobedo, Hernández, Estebané y Martínez, 2016). Dado los índices aportados por el AFC se infiere que, para la población estudiada, el constructo de EME, el modelo con dos variables latentes, culpa y orgullo, se comporta con un total de 12 variables observables (figura 1). Esto es: se confirma el modelo bifactorial propuesto y resuelto por el AFE, correspondientes a la emoción de culpa y de orgullo, donde cada factor integra los seis estadios morales. 
Tabla 6. Índices de ajuste del modelo bifactorial

\begin{tabular}{|l|l|l|l|l|l|l|l|}
\hline & RMSEA & GFI & CMIN/df & SRMR & CFI & NFI & TLI \\
\hline Esperado & $\approx 0.06$ & $>0.95$ & $<5.0$ & $\approx 0.05$ & $>0.95$ & $>0.95$ & $>0.95$ \\
\hline Encontrado & 0.054 & 0.961 & 1.874 & 0.0533 & 0.982 & 0.962 & 0.979 \\
\hline
\end{tabular}

Fuente: Elaboración propia

Figura 1. Modelo bifactorial del EEMO

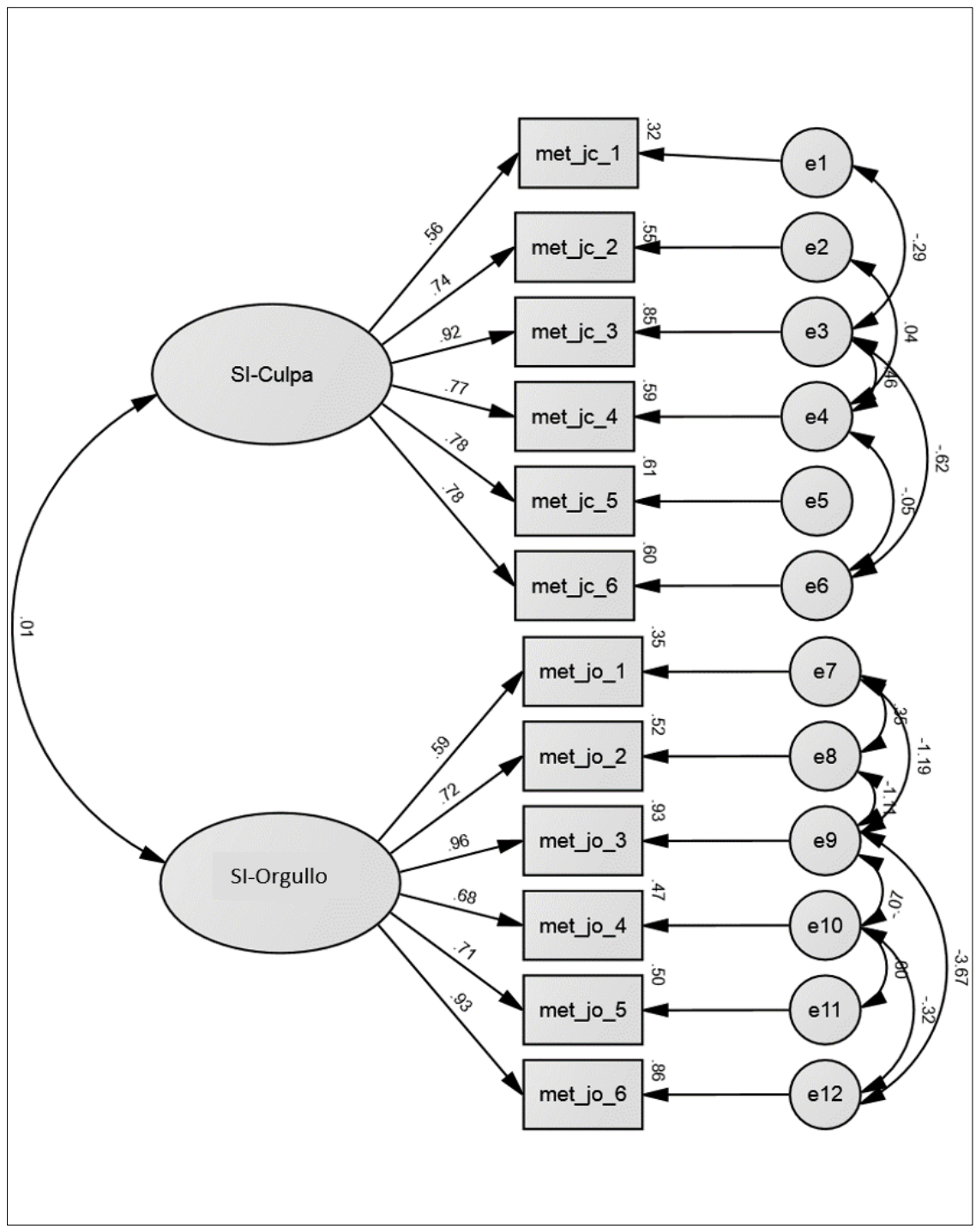

Fuente: Elaboración propia 


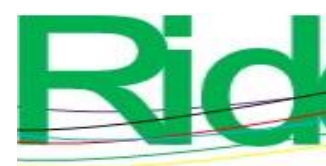

Revista Iberoamericana para la
Investigación y el Desarrollo Educativo
ISSN $2007-7467$

\section{Índice de confiabilidad}

Los valores de confiabilidad para los 12 ítems de la EEME del factor de culpa y el de orgullo fueron superiores a 0.80 , considerados como aceptables (tabla 7), ya que son superiores al mínimo (0.70) requerido (Nunnally, 1970).

Tabla 7. Confiabilidad del EMO y factores

\begin{tabular}{|l|l|l|}
\hline & $\begin{array}{l}\text { Alfa de } \\
\text { Cronbach }\end{array}$ & $\begin{array}{l}\text { Número } \\
\text { de ítems }\end{array}$ \\
\hline EME & 0.809 & 12 \\
\hline Culpa & 0.880 & 6 \\
\hline Orgullo & 0.883 & 6 \\
\hline
\end{tabular}

Fuente: Elaboración propia

\section{Validez convergente y discriminante}

Respecto a la evidencia de validez convergente, en la tabla 8 se observa que se encontraron correlaciones significativas y positivas. Los seis ítems de culpa tuvieron correlaciones positivas altas (entre 0.653 y 0.829 ) con el factor de culpa y los seis de orgullo igualmente correlacionaron altamente (entre 0.700 y 0.850 ) con el factor de orgullo. En relación con la evidencia de validez discriminante, se encontraron correlaciones negativas y débiles (de -0.036 a 0.080) entre los ítems de culpa y el factor de orgullo, de la misma forma que los seis ítems de orgullo correlacionaron negativa y débilmente con el de Culpa (de 0.103 a 0.154$)$. 
Tabla 8. Análisis de correlaciones de los ítems

\begin{tabular}{|c|c|c|}
\hline & Culpa & Orgullo \\
\hline Met-jc 1 & $0.653^{* *}$ & 0.037 \\
\hline Met-jc 2 & $0.823 * *$ & -0.020 \\
\hline Met-jc 3 & $0.844^{* *}$ & 0.021 \\
\hline Met-jc 4 & $0.800^{* *}$ & -0.036 \\
\hline Met-jc 5 & $0.829 * *$ & 0.080 \\
\hline Met-jc 6 & $0.793^{* *}$ & 0.047 \\
\hline Met-jo 1 & -0.103 & $0.700^{* *}$ \\
\hline Met-jo 2 & -0.042 & $0.795 * *$ \\
\hline Met-jo 3 & 0.056 & $0.781^{* *}$ \\
\hline Met-jo 4 & $0.154 * *$ & $0.814^{* *}$ \\
\hline Met-jo 5 & 0.053 & $0.850^{* *}$ \\
\hline Met-jo 6 & 0.010 & $0.828^{* *}$ \\
\hline
\end{tabular}

Fuente: Elaboración propia

\section{Discusión}

Pretendiendo estimular las investigaciones entre la moralidad y los factores emocionales, la presente investigación tuvo como objetivo validar y confirmar un modelo bifactorial correspondientes a la emoción de culpa y orgullo aplicando la EEME. Esto mediante un AFE y un AFC con muestras poblacionales independientes.

Los hallazgos del primer análisis (AFE) aportaron dos factores del modelo de EME, correspondientes a las emociones de culpa y orgullo. Ello ratificó los preceptos de Kaplan y Tivnan (2014) y Bericart (2012), entre otros autores, que apuntan a que el EME se explica entre una emoción dolorosa negativa y otra gratificante positiva. Esto es: evitar el dolor de la culpa y mantener el gozo del orgullo (Bericart, 2012; Vélez y Ostrosky, 2006). El análisis de componentes principales demostró un factor de Culpa con seis dimensiones y otro de orgullo con los respectivos seis estadios morales. Esto es: se demostró el modelo de EME con dos factores, cuya confiabilidad del alfa de Cronbach obtuvo valores aceptables y su validez convergente y discriminante fue buena, pues las correlaciones de los ítems de orgullo y de culpa fueron altas positivas, mientras que las correlaciones cruzadas fueron débiles negativas. 


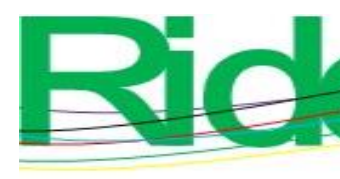

Revista Iberoamericana para la
Investigación y el Desarrollo Educativo
ISSN $2007-7467$

El segundo análisis (AFC), corroboró la estructura bifactorial del modelo del EME con el factor de culpa y el de orgullo, donde cada uno integró seis variables observables, respectivas a los seis estadios morales, haciendo un total de 12 ítems. En este análisis, la bondad de ajuste del modelo demostró que los índices fueron óptimos e indicaron un buen ajuste, conforme a Hu y Bentler (1999), Cangur y Ercan (2015) y Medrano y Muñoz (2017). Cabe señalar, además, que el modelo validado mostró covarianzas entre los errores de las variables observadas respectivas, por ejemplo, entre la tres y la cuatro, así como entre la siete y ocho. Se deduce que estas variaciones, a partir de lo expuesto por Kohlberg (1992), Kohlberg y Hersh (1977), se relacionan con los estadios contiguos y pertenecen al mismo nivel moral: el participante tendrá un sentido culposo o vergonzoso indistintamente en uno de los dos estadios del nivel moral correspondiente. En este sentido, como lo sugiere Lind (2008), no invalida el ajuste del modelo ni su escala respectiva.

\section{Conclusiones}

El modelo bifactorial de equilibrio moral, basado en las emociones de culpa y orgullo, junto con los seis estadios morales, fue validado y se observó un buen ajuste. Esto mediante un análisis exploratorio y uno confirmatorio, en dos momentos diferentes y con dos muestras respectivas. Asimismo, su escala correspondiente, para la población investigada de estudiantes universitarios, se constituyó como un instrumento eficaz para evaluar y diagnosticar el balance moral, así como una herramienta auxiliar para prevenir acciones desviadas, mejorar el equilibrio moral y emparejar el comportamiento con altos estándares morales o virtudes humanas.

Los resultados encontrados no se pueden generalizar a poblaciones diferentes a la abordada, pues fueron estudiantes universitarios quienes ayudaron a validar y ajustar el modelo bifactorial de EME. Si se desea ampliar la validez externa del modelo es necesario estudiar otras poblaciones, distintas a la estudiantil. 


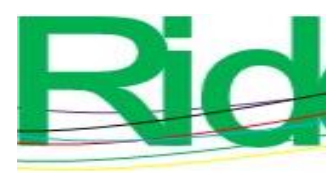

Revista Iberoamericana para la
Investigación y el Desarrollo Educativo
ISSN $2007-7467$

Futuras líneas de investigación

El presente artículo científico contribuye al desarrollo de la línea de investigación sobre la moral emocional. Se sugiere que el modelo de EME presentado funja como una herramienta de diagnóstico de las personas. Esto debido a que cuando ellas tienen un desbalance emocional, se sienten impulsadas a cometer acciones incorrectas, como actos de violencia, fraudes, abusos y comportamientos inmorales en general. De tal forma que, al diagnosticar el nivel de EME, se puede detectar alguna desviación conductual del sujeto y, una vez detectada, es posible prevenir funestas consecuencias individuales o colectivas.

Desde el ámbito de la salud emocional, una persona con un EME óptimo no sufre ningún trastorno. Contrariamente, el individuo con un desequilibrio negativo hacia un grado elevado de culpa sufrirá una sensación dolorosa. Inversamente, el sujeto con un nivel excesivo de orgullo genera en él un trastorno narcisista, una actitud de arrogancia y de menosprecio hacia los demás. Estos desequilibrios manifiestan trastornos en la salud emocional que precisan de algún tipo de intervención o terapia conductual.

Finalmente, futuras investigaciones deben orientarse hacia estrategias de afrontamiento para las personas con trastornos morales emocionales que establezcan pautas para incrementar las sensaciones gratificantes, emociones positivas e igualmente oriente el reconocimiento de lo punitivo de las negativas y así se optimice el EME. 


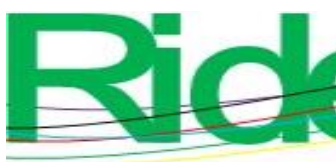

Revista Iberoamericana para la Investigación y el Desarrollo Educativo ISSN 2007-7467

\section{Referencias}

Bericart, E. (2012). Emociones. Sociopedia.isa, 1-13.

Beruchashvili, M., Price, L. and Gentry, J. W. (2006). Striving to be Good: Moral Balance in Consumer Choice. In Ekstrom, K. M. and Brembeck, H. (eds.), European Advances in Consumer Research (pp. 303-308). Goteborg, Sweden: Association for Consumer Research.

Bush, S. (2013). Horribly Wrong: Moral Disgust and Killing. Journal Religious Ethics, 41(4), 585-600. Retrieved from https://onlinelibrary.wiley.com/doi/abs/10.1111/jore.12033.

Cangur, S. and Ercan, I. (2015). Comparison of model fit indices used in structural equation modeling under multivariate normality. Journal of Modern Applied Statistical Methods, 14(1), 152-167. Retrieved from https://doi.org/10.22237/jmasm/1430453580.

Caparrós, L. (1986). Desbordamiento de la personalidad y horror moral en La gran Semíramis, de Virués. Epos, (2), 49-52. Recuperado de https://doi.org/10.5944/epos.2.1986.9463.

Chión, S. and Charles, V. (2016). Analítica de datos para la modelación estructural. México: Pearson.

Cova, F., Deonna, J. and Sander, D. (2015). Introduction: Moral Emotions. Topoi, 34(2), 397-400. Retrieved from https://doi.org/10.1007/s11245-015-9345-0.

Cupani, M. (2012). Análisis de ecuaciones estructurales: conceptos, etapas de desarrollo y un ejemplo de aplicación. Revista Tesis, (1), 186-199. http://www.revistas.unc.edu.ar/index.php/tesis/article/download/2884/2750.

Erlandsson, A. (2006). The differences in Frequent and Intense Affect Balance when measuring Subjective Well-Being and Personality. (Thesis advanced). Växjö Universitet, Växjö. Retrieved from http://urn.kb.se/resolve?urn=urn:nbn:se:vxu:diva773.

Escobedo, M., Hernández, J., Estebané, V. y Martínez, G. (2016). Modelos de ecuaciones estructurales: Características, fases, construcción, aplicación y resultados. Ciencia \& Trabajo, 18(55), 16-22. Recuperado de https://doi.org/10.4067/s071824492016000100004. 


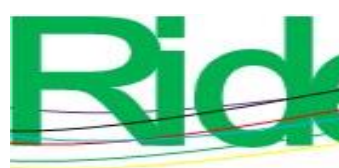

\section{Revista Iberoamericana para la Investigación y el Desarrollo Educativo ISSN $2007-7467$}

Etikan, I., Abubakar, S. and Sunusi, R. (2016). Comparison of Convenience Sampling and Purposive Sampling. American Journal of Theoretical and Applied Statistics, 5(1), 14. Retrieved from https://doi. org/110.11648/j.ajtas.20160501.11.

Ferrando, P. y Anguiano, C. (2010). El análisis factorial como técnica de investigación en psicología. Papeles del Psicólogo, 31(1), 18-33. Recuperado de http:// www.papelesdelpsicologo.es/pdf/1793.pdf.

Freud, S. (1923). Das Ich und das Es. Leipzig, Deutschland: Internationaler Psychoanalytischer Verlag.

Greene, J., and Haidt, J. (2002). How (and where) does moral judgment work? Trends in Cognitive Sciences, 6(12), 517-523. doi:10.1016/S1364-6613(02)02011-9

Hair, J., Black, W., Babin, B. and Anderson, R. (2010). Multivariate Data Analysis: A Global Perspective. Harlow, England: Pearson.

Hernández, R., Fernández, C. y Baptista, P. (2014). Metodología de la investigación. Ciudad de México, México: McGraw-Hill.

Horne, Z. and Powell, D. (2016). How Large Is the Role of Emotion in Judgments of Moral Dilemmas? PLoS One, 11(7), 1-19. Retrieved from https://pubmed.ncbi.nlm.nih.gov/27385365/.

$\mathrm{Hu}, \mathrm{L}$. and Bentler, P. (1999). Cutoff criteria for fit indexes in covariance structure analysis: Conventional criteria versus new alternatives. Structural Equation Modeling: A Multidisciplinary Journal, 6(1), 1-55. Retrieved from https://doi.org/10.1080/10705519909540118.

Kaplan, U. and Tivnan, T. (2014). Multiplicity of Emotions in Moral Judgment and Motivation. Ethics and Behavior, 24(6), 421-443. Retrieved from https://doi.org/10.1080/10508422.2014.888517.

Kohlberg, L. (1992). Psicología del desarrollo moral. Bilbao, España: Desclée de Brouwer. Kohlberg, L. and Hersh, R. (1977). Moral Development: A Review of the Theory. Theory into Practice, 16(2), 53-59. Retrieved from http://links.jstor.org/sici?sici=0040$5841 \% 28197704 \% 2916 \% 3 \mathrm{~A} 2 \% 3 \mathrm{C} 53 \% 3 \mathrm{AMDAROT} \% 3 \mathrm{E} 2.0 . \mathrm{CO} \% 3 \mathrm{~B} 2-\% 23$.

Lind, G. (2008). The meaning and measurement of moral judgment competence revisited. A dual aspect model. In Fasko, D. and Willis, W. (eds.), Contemporary Philosophical and Psychological Perspectives on Moral Development and Education (pp.185-220). New Jersey, United States: Hampton Press. 


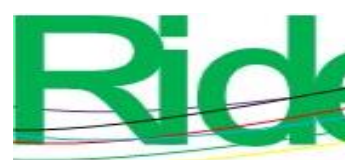

Revista Iberoamericana para la
Investigación y el Desarrollo Educativo
ISSN $2007-7467$

Lindenbaum, D., Geddes, D. and Gabriel, Y. (2017). Moral Emotions and Ethics in Organisations: Introduction to the Special Issue. Journal Business Ethics, 141(4), 645-656. Retrieved from https://doi.org/10.1007/s10551-016-3201-z.

Malti, T., Ongley, F., Dys, S. and Colasante, T. (2012). Adolescents' emotions and reasoning in contexts of moral conflict and social exclusion. New Directions For Youth Development, $\quad$ (136), $27 . \quad$ Retrieved from https://ezproxy.upaep.mx:2107/10.1002/yd.20036.

Medrano, L. A. y Muñoz, R. (2017). Aproximación conceptual y práctica a los modelos de ecuaciones estructurales. Revista Digital de Investigación en Docencia Universitaria, 11(1), 219-239. Recuperado de https://doi.org/10.19083/ridu.11.486.

Nisan, M. (1991). The Moral Balance Model: Theory and Research. Extendending our Understanding of Moral Choice and Deviation. In Kurtines, W. and Gewirtz, J. (ed.), Handbook of Moral Behavior and Development (pp. 213-249). New Jersey, United States: Lawrence Erlbaum Associates, Publishers

Nunnally, J. (1970). Introduction to Psychological Measurement. New York, United States: McGraw-Hill.

Orsi, R. (2006). Emociones morales y moralidad. Ideas y valores. Ideas y Valores, 55(131), 33-49.

Prinz J. (2006). The emotional basis of moral judgments. Philosophical Explorations, 9(1), $29-43$. Retrieved from https://www.tandfonline.com/doi/abs/10.1080/13869790500492466.

Prinz, J. (2007). Can Moral Obligations Be Empirically Discovered? Midwest Studies in Philosophy, 31, 271-291. Retrieved from https://onlinelibrary.wiley.com/doi/abs/10.1111/j.1475-4975.2007.00148.x.

Pinedo, I. (2015). El factor emocional en la construcción del juicio moral: una trayectoria desde Kohlberg al horizonte de la filosofía experimental y la neurociencia cognitiva. Revista Interdisciplinaria de Filosofía y Psicología, 10(32), 15-25.

Rebega, O. (2017). Gender Differences in Moral Concerns, Guilt and Shame Proness, and Empathy. Romanian Journal of Experimental Applied Psychology, (8), 130-135. Retrieved from https://www.rjeap.ro/volume-8-special-issue-1-2017-psiworld-2016proceedings/rjeap/volume-8-special-issue-1-2017-psiworld-2016-proceedings/20paper-30-gender-differences-in-moral-concerns-guilt-and-shame-proness-andempathy. 

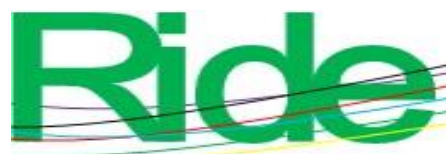

Revista Iberoamericana para la
Investigación y el Desarrollo Educativo
ISSN $2007-7467$

Robles, V. (2019). Introducing the Emotive Moral Competence Index (EMCI): A New Measurement of Moral Judgment. Iranian Journal of Psychiatry and Behavioral Sciences, 13(4).

Santoya, Y., Garcés, M. y Tezón, M. (2018). Las emociones en la vida universitaria: análisis de la relación entre autoconocimiento emocional y autorregulación emocional en adolescentes y jóvenes universitarios. Psicogente, 21(40), 124-137. Recuperado de https://doi.org/10.17081/psico.21.40.3081.

Tangney, J. P., Stuewig, J. and Mashek, D. J. (2007). Moral Emotions and Moral Behavior. Annual Review of Psychology, 58, 345-372. Retrieved from https://doi.org/10.1146/annurev.psych.56.091103.070145.

Vélez, A. and Ostrosky, F. (2006). From morality to moral emotions. International Journal of Psychology, 41(5), 348-354. Retrieved from https://www.tandfonline.com/doi/abs/10.1080/00207590500345898. 


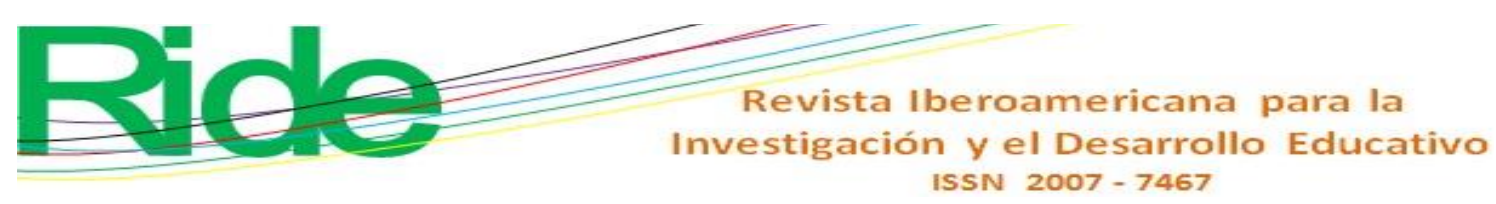

Anexo 1. Escala de Equilibrio Moral Emocional (EEMO)

Instrucciones:

A continuación, encontrarás una historia. Léela con detenimiento, observando e imaginando los detalles expresados en ella. Una vez leída y comprendida la historia, encontrarás una pregunta sobre qué tan horrible te pareció la historia. Posteriormente, encontrarás seis enunciados sobre la culpa del protagonista de la historia y seis sobre el orgullo. Cada enunciado tiene que ser valorado por ti en una escala del 1 al 10. Por favor, señala con una $X$ solo un número de la escala.

HISTORIA DE JUAN

Una noche muy lluviosa, con oscuridad profunda y alta dificultad para observar personas y baches, en un barrio de la zona conurbada de la Ciudad de México, Juan regresaba de su trabajo, cansado de su jornada y de manejar por más de una hora su automóvil hasta su casa. Una vez habiendo detenido su auto enfrente de su hogar, Juan desciende para abrir la puerta de su cochera. En ese mismo instante, un hombre, cubierto el rostro, complexión ligeramente alta y robusta, con pistola en mano, sigilosamente se acerca para despojarlo de sus pertenencias personales y de su auto.

Juan siempre en momentos angustiantes graves recordaba a su Sra. madre. Abnegada, dedicada e inteligente, le había inculcado principios y valores como el amor a la vida, la sabiduría y la prudencia. Su madre, a pesar de ser ultrajada, dio a luz a su hijo producto de tal vejación, Juan. Sin embargo, por su buena educación, Juan, soltero aún, respetaba y valoraba el recuerdo de su padre, a quien nunca conoció.

En el transcurso del asalto, al despojarse de su reloj, anillo, celular, cartera y llaves, súbitamente, en un descuido del asaltante, Juan, con destreza magistral, experimentado en la defensa personal por más de ocho años, elude e invierte el arma, con la misma mano del asaltante en su contra, disparándose al mismo momento el arma de fuego. El asaltante sale huyendo, mientras Juan, asustado y angustiado, observando una mancha de sangre en su ropa, entra a su casa... ¡No tiene absolutamente nada! Y da cuentas de lo que sucedió a su querida mamá, se cambia de ropa y se retira a descansar. Sin embargo, durante gran parte de la noche, no puede descansar, sobresaltándose y sufriendo pesadillas de aquel hombre encapuchado, quizás mal herido.

Ya entrada la madrugada, Juan concilia el sueño... Empero, a escasas dos horas, su mamá, aturdida, entra bruscamente a su recamara y con periódico en mano le da la noticia: que en el 


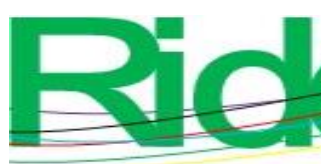

e

Revista Iberoamericana para la

Investigación y el Desarrollo Educativo

ISSN 2007 - 7467

barrio había muerto una persona a unas cuadras de su casa, de un disparo, y su foto estaba en primera plana. Después de enseñarle el diario a Juan, donde yacía el rostro e imagen de aquel cadáver, ella le expresa asombrada y horrorizada:

— ¿Ese es ... era, tu padre!

¿Qué tan horrible siente tú lo que hizo Juan?

$\begin{array}{llllllllllll}\text { Absolutamente nada horrible } & 1 & 2 & 3 & 4 & 5 & 6 & 7 & 8 & 9 & 10 & \text { Totalmente horrible }\end{array}$

Si tú fueras Juan, ¿con qué grado sentirías culpa por matar a

tu padre? Considera las siguientes razones:

1) Solo por quitarle la vida a alguien y quizás por

$\begin{array}{llllllllll}1 & 2 & 3 & 4 & 5 & 6 & 7 & 8 & 9 & 10\end{array}$

defenderme vaya a prisión.

2) Por matar a mi padre, yo no podría ver a mi madre a los

$\begin{array}{llllllllll}1 & 2 & 3 & 4 & 5 & 6 & 7 & 8 & 9 & 10\end{array}$ ojos.

3) Por matar a mi padre me señalarían, además de mi

$\begin{array}{llllllllll}1 & 2 & 3 & 4 & 5 & 6 & 7 & 8 & 9 & 10\end{array}$ madre, toda mi familia, mi novia y amigos.

4) Porque me atormentaría imaginar que las personas que

$\begin{array}{llllllllll}1 & 2 & 3 & 4 & 5 & 6 & 7 & 8 & 9 & 10\end{array}$ más amo no podrán vivir en armonía en sociedad y menos junto a mí por mi culpa.

5) Que traería el rechazo de la gente y de toda lo sociedad

$\begin{array}{llllllllll}1 & 2 & 3 & 4 & 5 & 6 & 7 & 8 & 9 & 10\end{array}$ por ser el asesino de mi padre.

6) Porque no cumplí el principio inculcado por mi madre, el

$\begin{array}{llllllllll}1 & 2 & 3 & 4 & 5 & 6 & 7 & 8 & 9 & 10\end{array}$ amor a la vida, la prudencia y el respeto hacia el padre.

Si tú fueras Juan, ¿con qué grado sentirías orgullo por matar a tu padre? Considera las siguientes razones:

7) Por defender mi vida y emplear lo que aprendí de

$\begin{array}{llllllllll}1 & 2 & 3 & 4 & 5 & 6 & 7 & 8 & 9 & 10\end{array}$ defensa personal.

8) Por defenderme bien, cuidar de mí, como me lo enseñó mi mamá.

9) Por matar a mi padre, quien no se ocupó de mí nunca y

$\begin{array}{llllllllll}1 & 2 & 3 & 4 & 5 & 6 & 7 & 8 & 9 & 10\end{array}$ violó a mi mamá, que siendo ladrón tendría la aprobación de la gente y cualquiera actuaría de la misma forma.

$\begin{array}{llllllllll}1 & 2 & 3 & 4 & 5 & 6 & 7 & 8 & 9 & 10\end{array}$ 

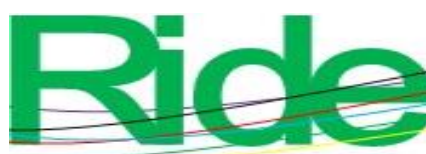

Revista Iberoamericana para la Investigación y el Desarrollo Educativo ISSN 2007 - 7467

10) Porque aparte de mi madre, toda mi familia y la sociedad

$\begin{array}{llllllllll}1 & 2 & 3 & 4 & 5 & 6 & 7 & 8 & 9 & 10\end{array}$ me reconocerían.

11) Que traería el reconocimiento de la gente y de toda lo

$\begin{array}{llllllllll}1 & 2 & 3 & 4 & 5 & 6 & 7 & 8 & 9 & 10\end{array}$ sociedad por castigar a alguien que no cumple con la reglas sociales ni humanas.

12) Porque cumplí el principio de salvaguardar mi vida,

$\begin{array}{llllllllll}1 & 2 & 3 & 4 & 5 & 6 & 7 & 8 & 9 & 10\end{array}$ principio inculcado por mi madre, el de justicia y el respeto del deber 